\title{
A Table of Elliptic Integrals: Cubic Cases*
}

\author{
By B. C. Carlson
}

\begin{abstract}
Forty-one integrands that are rational except for the square root of a cubic polynomial with known real zeros are integrated in terms of $R$-functions for which Fortran codes are available. In contrast to conventional tables the interval of integration is not required to begin or end at a singular point of the integrand. The table contains one elliptic integral of the first kind, 26 of the second kind, and 14 of the third kind. Only 10 of the integrals are treated in standard tables, which list a large number of special cases that are unified here.
\end{abstract}

1. Introduction. Two earlier installments [2], [3] of a new table of elliptic integrals deal primarily with "quartic cases" in which the integrand is rational except for the square root of a quartic polynomial with known real zeros. In this paper we consider "cubic cases" of the form

$$
[p]=\left[p_{1}, \ldots, p_{4}\right]=\int_{y}^{x} \prod_{i=1}^{4}\left(a_{i}+b_{i} t\right)^{p_{i} / 2} d t
$$

where $p_{1}, p_{2}, p_{3}$ are odd integers, $p_{4}$ is an even integer (omitted if it is zero), and all quantities are real. Integrands containing complex conjugate factors will be considered in a later paper. Although a cubic case can usually be calculated numerically by choosing $a_{i}=1$ and $b_{i}=0$ for some value of $i$ in a suitable quartic case, it is preferable to have an explicit formula, which is often tedious to obtain in a uniform notation from the quartic case.

The integral (1.1) is an elliptic integral of the third kind if $p_{4}$ is even and negative. Otherwise, it is second kind except for $[-1,-1,-1]$, which is first kind. Many integrals like

$$
\int\left(\alpha \cos ^{2} \theta+\beta \sin ^{2} \theta\right)^{p_{1} / 2} d \theta \text { and } \int\left(\alpha+\beta z^{2}\right)^{p_{1} / 2}\left(\gamma+\delta z^{2}\right)^{p_{2} / 2} d z
$$

can be put in the form (1.1) by letting $t=\sin ^{2} \theta$ or $t=z^{2}$.

The integrals in the table are expressed in terms of four $R$-functions:

$$
\begin{gathered}
R_{F}(x, y, z)=\frac{1}{2} \int_{0}^{\infty}[(t+x)(t+y)(t+z)]^{-1 / 2} d t, \\
R_{J}(x, y, z, w)=\frac{3}{2} \int_{0}^{\infty}[(t+x)(t+y)(t+z)]^{-1 / 2}(t+w)^{-1} d t,
\end{gathered}
$$

Received July 8, 1988.

1980 Mathematics Subject Classification (1985 Revision). Primary 33A25; Secondary 33A30.

*Part of this work was done at the University of Maryland, where the author was a visitor at the Institute for Physical Science and Technology, with the support of AROD contract DAAG 2980-C-0032. The rest was done in the Ames Laboratory, which is operated for the U. S. Department of Energy by Iowa State University under contract no. W-7405-ENG-82. The work was supported by the Director of Energy Research, Office of Basic Energy Sciences. 
and two special cases,

$$
R_{C}(x, y)=R_{F}(x, y, y) \quad \text { and } \quad R_{D}(x, y, z)=R_{J}(x, y, z, z) .
$$

The functions $R_{F}$ and $R_{J}$ are symmetric in $x, y, z ; R_{F}$ and $R_{C}$ are homogeneous of degree $-1 / 2 ; R_{J}$ and $R_{D}$ are homogeneous of degree $-3 / 2$; each of the four functions has the value unity when all its arguments are unity; and $R_{C}$ and $R_{J}$ are interpreted as Cauchy principal values when the last argument is negative. Fortran codes for numerical computation of all four functions, including Cauchy principal values, are listed in the Supplements to [2] and [3]. The functions $R_{F}, R_{D}$, and $R_{J}$ respectively replace Legendre's integrals of the first, second, and third kinds, while $R_{C}$ includes the inverse circular and inverse hyperbolic functions.

To select integrals that are relatively simple, we arbitrarily require $\sum\left|p_{i}\right| \leq 7$ and $\sum p_{i} \leq 3$. Apart from permutation of subscripts in (1.1), there are just 40 cases of this kind. The table in Section 2 contains all 40 as well as $[-3,-3,-3]$, while only 10 of the 41 are included in [4] and nine in [5]. Each of the formulas for $[1,-1,-1],[1,1,-1],[-1,-1,-3]$, and $[-1,-1,-5]$ unifies 18 formulas in [4], and that for $[1,-1,-3]$ unifies 36. Moreover, the table in Section 2 does not require the interval of integration to begin or end at a singular point of the integrand.

Derivation of the formulas is discussed in Section 3. All integral formulas have been checked by numerical integration, and some details of the checks are given in Section 4.

2. Table of Cubic Cases. We assume $x>y$ and $a_{i}+b_{i} t>0, y<t<x$, for $i=1,2,3$. Assumptions about $a_{4}+b_{4} t$ will be stated where necessary. We define

$$
\begin{gathered}
d_{i j}=a_{i} b_{j}-a_{j} b_{i}, \quad r_{i j}=\frac{d_{i j}}{b_{i} b_{j}}=\frac{a_{i}}{b_{i}}-\frac{a_{j}}{b_{j}} \\
X_{i}=\left(a_{i}+b_{i} x\right)^{1 / 2}, \quad Y_{i}=\left(a_{i}+b_{i} y\right)^{1 / 2} \\
U_{i}=\left(X_{i} Y_{j} Y_{k}+Y_{i} X_{j} X_{k}\right) /(x-y),
\end{gathered}
$$

where $i, j, k$ is any permutation of $1,2,3$;

$$
\begin{gathered}
W_{2}^{2}=U_{1}^{2}-b_{4} d_{12} d_{13} / d_{14} \\
Q_{2}^{2}=\left(X_{4} Y_{4} W_{2} / X_{1} Y_{1}\right)^{2}, \quad P_{2}^{2}=Q_{2}^{2}+b_{4} d_{24} d_{34} / d_{14} \\
A\left(p_{1}, \ldots, p_{n}\right)=X_{1}^{p_{1}} \cdots X_{n}^{p_{n}}-Y_{1}^{p_{1}} \cdots Y_{n}^{p_{n}}
\end{gathered}
$$

These definitions imply, if $P_{2}$ is chosen positive,

$$
\begin{gathered}
P_{2}=\left(X_{1}^{-1} X_{2} X_{3} Y_{4}^{2}+Y_{1}^{-1} Y_{2} Y_{3} X_{4}^{2}\right) /(x-y), \\
U_{2}^{2}=U_{1}^{2}-b_{3} d_{12}, \quad U_{3}^{2}=U_{1}^{2}-b_{2} d_{13}=U_{2}^{2}-b_{1} d_{23}, \\
W_{2}^{2}=U_{2}^{2}+b_{1} d_{12} d_{34} / d_{14}=U_{3}^{2}+b_{1} d_{13} d_{24} / d_{14} .
\end{gathered}
$$

If one limit of integration is infinite, (2.3) simplifies to

$$
\begin{aligned}
& U_{i}=\left(b_{j} b_{k}\right)^{1 / 2} Y_{i}, \quad x=+\infty, \\
& U_{i}=\left(b_{j} b_{k}\right)^{1 / 2} X_{i}, \quad y=-\infty,
\end{aligned}
$$

where the square roots are nonnegative, while

$$
\begin{array}{ll}
Q_{2}^{2}=\left(b_{4} / b_{1}\right)\left(Y_{4} W_{2} / Y_{1}\right)^{2}, & x=+\infty \\
Q_{2}^{2}=\left(b_{4} / b_{1}\right)\left(X_{4} W_{2} / X_{1}\right)^{2}, & y=-\infty
\end{array}
$$


Cubic cases will be expressed in terms of the quantities

$$
\begin{gathered}
I_{1 c}=2 R_{F}\left(U_{3}^{2}, U_{2}^{2}, U_{1}^{2}\right), \\
I_{2 c}=\frac{2}{3} d_{12} d_{13} R_{D}\left(U_{3}^{2}, U_{2}^{2}, U_{1}^{2}\right)+2 X_{1} Y_{1} / U_{1}, \\
I_{3 c}=\frac{-2 b_{1} d_{12} d_{13}}{3 d_{14}} R_{J}\left(U_{3}^{2}, U_{2}^{2}, U_{1}^{2}, W_{2}^{2}\right)+2 R_{C}\left(P_{2}^{2}, Q_{2}^{2}\right),
\end{gathered}
$$

which are integrals of the first, second, and third kinds, respectively. It will be seen from the tables that

$$
I_{1 c}=[-1,-1,-1], \quad I_{2 c}=[1,-1,-1], \quad I_{3 c}=[1,-1,-1,-2] .
$$

Thus $I_{3 c}$ reduces to $I_{2 c}$ if $a_{4}=1$ and $b_{4}=0$. The extra subscript $c$ stands for "cubic," and the quantities defined here are obtained from those used in [3] for quartic cases. Specifically, if we put $a_{4}=1$ and $b_{4}=0$ and subsequently replace the subscript 5 by 4 , then $\left(U_{12}, U_{13}, U_{14}\right)$ reduces to $\left(U_{3}, U_{2}, U_{1}\right),(W, P, Q)$ to $\left(W_{2}, P_{2}, Q_{2}\right)$, and $\left(I_{1}, I_{2}, I_{3}, I_{3}^{\prime}\right)$ to $\left(I_{1 c}, I_{2 c}, I_{3 c}, I_{2 c}\right)$.

It is convenient to define also the quantities

$$
\begin{aligned}
J_{1 c} & =d_{12} d_{13} I_{1 c}-2 b_{1} A(1,1,1) \\
& =2 d_{12} d_{13} R_{F}\left(U_{3}^{2}, U_{2}^{2}, U_{1}^{2}\right)-2 b_{1} A(1,1,1), \\
J_{2 c} & =b_{2} I_{2 c}-2 A(1,1,-1) \\
& =\frac{2}{3} b_{2} d_{12} d_{13} R_{D}\left(U_{3}^{2}, U_{2}^{2}, U_{1}^{2}\right)+\frac{2 d_{13} X_{2} Y_{2}}{X_{3} Y_{3} U_{1}} .
\end{aligned}
$$

The first of these appears in the formula for $[1,1,-1]$ and is transmitted by recurrence relations to a dozen others; likewise, $J_{2 c}$ is transmitted from $[1,-1,-3]$. The second line of $(2.17)$ follows from the first with the help of the identity

$$
\begin{aligned}
(x-y) U_{1} X_{3} Y_{3} A(1,1,-1) & =X_{2} Y_{2}\left(X_{1}^{2} Y_{3}^{2}-X_{3}^{2} Y_{1}^{2}\right)+X_{1} X_{3} Y_{1} Y_{3}\left(X_{2}^{2}-Y_{2}^{2}\right) \\
& =(x-y)\left(d_{31} X_{2} Y_{2}+b_{2} X_{1} X_{3} Y_{1} Y_{3}\right)
\end{aligned}
$$

It is important to use $I_{1 c}$ and $J_{2 c}$, not $J_{1 c}$ or $I_{2 c}$, to evaluate integrals with $\sum p_{i}<$ -2 , which converge when $x=+\infty$ or $y=-\infty$. Both $J_{1 c}$ and $I_{2 c}$ then become infinite while $I_{1 c}$ and $J_{2 c}$ are finite. The second term in the second line of $(2.17)$ becomes

$$
\begin{array}{ll}
2 d_{13} X_{2} Y_{2} / X_{3} Y_{3} U_{1}=2 d_{13} Y_{2} / b_{3} Y_{1} Y_{3}, & x=+\infty \\
2 d_{13} X_{2} Y_{2} / X_{3} Y_{3} U_{1}=-2 d_{13} X_{2} / b_{3} X_{1} X_{3}, & y=-\infty
\end{array}
$$

If one limit of integration is a branch point of the integrand, then $X_{i}$ or $Y_{i}$ is 0 for some value of $i \leq 3$, and one of the two terms on the right-hand side of (2.3) vanishes. If $X_{1} Y_{1}=0$ then $P_{2}$ and $Q_{2}$ are infinite, and the $R_{C}$ function in (2.14) vanishes by homogeneity. If both limits of integration are branch points, the elliptic integral is called complete, and $U_{1} U_{2} U_{3}=0$. It is not assumed that $b_{i} \neq 0$ nor that $d_{i j} \neq 0$ unless one of these quantities occurs in a denominator. The relation $d_{i j}=0$ is equivalent to proportionality of $a_{i}+b_{i} t$ and $a_{j}+b_{j} t$.

We shall now list 41 cases of

$$
\left[p_{1}, \ldots, p_{4}\right]=\int_{y}^{x}\left(a_{1}+b_{1} t\right)^{p_{1} / 2} \cdots\left(a_{4}+b_{4} t\right)^{p_{4} / 2} d t
$$


18 with $p_{4}=0$, nine with $p_{4}=2$ or 4 , and 14 with $p_{4}=-2$ or -4 . Only the last 14 are integrals of the third kind involving $I_{3 c}$. We omit $p_{4}=0$ in the first 18:

(2.21) $[-1,-1,-1]=I_{1 c}$.

(2.22) $[1,-1,-1]=I_{2 c}$.

(2.23) $[1,1,-1]=\left[\left(b_{1} d_{23}+b_{2} d_{13}\right) I_{2 c}-J_{1 c}\right] / 3 b_{1} b_{3}$.

(2.24) $[1,1,1]=\left[-2 b_{1} b_{2} b_{3}\left(r_{12} r_{13}+r_{23}^{2}\right) I_{2 c}+\left(r_{12}+r_{13}\right) J_{1 c}+6 A(3,1,1)\right] / 15 b_{1}$.

(2.25) $[1,-1,-3]=\left(J_{2 c}-d_{12} I_{1 c}\right) / d_{23}$.

(2.26) $[-1,-1,-3]=\left(b_{3} J_{2 c}-b_{2} d_{13} I_{1 c}\right) / d_{13} d_{23}$.

(2.27) $[1,1,-3]=\left[2 b_{2} I_{2 c}-d_{12} I_{1 c}-2 A(1,1,-1)\right] / b_{3}$.

(2.28) $[3,-1,-1]=\left[2\left(b_{2} d_{13}+b_{3} d_{12}\right) I_{2 c}-J_{1 c}\right] / 3 b_{2} b_{3}$.

(2.29) $[3,1,-1]=\left[b_{1} b_{2} b_{3}\left(3 r_{13}^{2}+7 r_{13} r_{23}-2 r_{23}^{2}\right) I_{2 c}\right.$ $\left.-\left(3 r_{13}+r_{23}\right) J_{1 c}+6 A(3,1,1)\right] / 15 b_{3}$.

(2.30) $[3,-1,-3]=\left[\left(b_{1} d_{23}+b_{2} d_{13}\right) I_{2 c}-d_{12} d_{13} I_{1 c}-2 d_{13} A(1,1,-1)\right] / b_{3} d_{23}$.

$$
\begin{gathered}
{[3,1,-3]=\left[\left(b_{1} d_{23}+7 b_{2} d_{13}\right) I_{2 c}-4 d_{12} d_{13} I_{1 c}\right.} \\
\left.+2 b_{1} A(1,1,1)-6 d_{13} A(1,1,-1)\right] / 3 b_{3}^{2} . \\
{[1,-3,-3]=\left[2 b_{3} J_{2 c}-\left(b_{2} d_{13}+b_{3} d_{12}\right) I_{1 c}+2 d_{23} A(1,-1,-1)\right] / d_{23}^{2} .} \\
{[-1,-3,-3]=\left[b_{3}\left(b_{2} d_{13}+b_{3} d_{12}\right) J_{2 c}-2 b_{2} b_{3} d_{12} d_{13} I_{1 c}\right.} \\
\left.+2 b_{2} d_{13} d_{23} A(1,-1,-1)\right] / d_{12} d_{13} d_{23}^{2} . \\
{[-3,-3,-3] \quad \begin{array}{c}
\left.+\left(2 / r_{12}\right)\left[b_{1} r_{23} A(-1,1,-1)+b_{2} r_{13} A(1,-1,-1)\right]\right\} \\
=\left(b_{3} / d_{12} d_{13} d_{23}\right)\left\{\left(2 / r_{12} r_{13} r_{23}\right)\left(r_{12} r_{13}+r_{23}^{2}\right) J_{2 c}\right.
\end{array}} \\
-\left(b_{1} b_{2} / r_{23}\right)\left(r_{12}+r_{13}\right) I_{1 c} \\
{[1,-1,-5]=\left[-b_{2}\left(1+r_{12} / r_{13}\right) J_{2 c}+2 b_{2} d_{12} I_{1 c}-2 d_{23} A(1,1,-3)\right] / 3 d_{23}^{2} .} \\
{[1,1,-5]=\left[\left(r_{13}^{-1}+r_{23}^{-1}\right) J_{2 c}-d_{12} r_{23}^{-1} I_{1 c}-2 b_{3} A(1,1,-3)\right] / 3 b_{3}^{2} .} \\
{[-1,-1,-5]=\left[-2\left(r_{13}^{-1}+r_{23}^{-1}\right) J_{2 c}+b_{1} b_{2}\left(1+2 r_{13} / r_{23}\right) I_{1 c}\right.} \\
\left.-2 b_{3} A(1,1,-3)\right] / 3 d_{13} d_{23} . \\
{[5,-1,-1]=\left[b_{1}^{2} b_{2} b_{3}\left(8 r_{12}^{2}+8 r_{13}^{2}+7 r_{12} r_{13}\right) I_{2 c}\right.} \\
\left.-4 b_{1}\left(r_{12}+r_{13}\right) J_{1 c}+6 b_{1} A(3,1,1)\right] / 15 b_{2} b_{3} .
\end{gathered}
$$

The next nine integrals have $p_{4}=2$ or 4 . No restriction is placed on $a_{4}$ or $b_{4}$.

(2.39) $[-1,-1,-1,2]=\left(b_{4} I_{2 c}-d_{14} I_{1 c}\right) / b_{1}$.

(2.40) $[1,-1,-1,2]=\left(b_{4} / 3\right)\left[\left(r_{13}-r_{34}-2 r_{24}\right) I_{2 c}-J_{1 c} / b_{1} b_{2} b_{3}\right]$.

$$
\begin{array}{r}
{[1,1,-1,2]=\left(b_{4} / 15 b_{1} b_{3}\right)\left\{-b_{1} b_{2} b_{3}\left[r_{12}^{2}+r_{13}^{2}+r_{23}^{2}+5 r_{34}\left(r_{13}+r_{23}\right)\right] I_{2 c}\right.} \\
\left.+\left(r_{12}+r_{14}+4 r_{34}\right) J_{1 c}+6 A(3,1,1)\right\} .
\end{array}
$$

(2.42) $[-1,-1,-3,2]=\left(-d_{34} J_{2 c}+d_{13} d_{24} I_{1 c}\right) / d_{13} d_{23}$. 


$$
\begin{gathered}
{[1,-1,-3,2]=\left[\left(b_{4} d_{23}-b_{2} d_{34}\right) I_{2 c}+d_{12} d_{34} I_{1 c}+2 d_{34} A(1,1,-1)\right] / b_{3} d_{23} .} \\
\begin{array}{c}
{[1,1,-3,2]=\left(b_{4} / 3 b_{3}\right)\left[b_{2}\left(r_{13}+r_{23}-6 r_{34}\right) I_{2 c}+d_{12}\left(3 r_{34}-r_{13}\right) I_{1 c}\right.} \\
\left.+\left(2 / b_{3}\right) A(1,1,1)+6 r_{34} A(1,1,-1)\right] . \\
{[3,-1,-1,2]=\left(b_{4} / 15 b_{2} b_{3}\right)\left\{b _ { 1 } b _ { 2 } b _ { 3 } \left[8 r_{12}^{2}+8 r_{13}^{2}+7 r_{12} r_{13}\right.\right.} \\
\left.-10 r_{14}\left(r_{12}+r_{13}\right)\right] I_{2 c} \\
\left.-\left(4 r_{12}+4 r_{13}-5 r_{14}\right) J_{1 c}+6 A(3,1,1)\right\} . \\
{[-1,-1,-1,4]=\left(b_{4}^{2} / 3 b_{1}\right)\left[-2\left(r_{14}+r_{24}+r_{34}\right) I_{2 c}\right.} \\
\left.+3 b_{1} r_{14}^{2} I_{1 c}-J_{1 c} / b_{1} b_{2} b_{3}\right] . \\
{[1,-1,-1,4]=\left(b_{4}^{2} / 15 b_{1} b_{2} b_{3}\right)\left\{b_{1} b_{2} b_{3}\left(8 r_{23}^{2}+3 r_{12} r_{13}-5 r_{14}^{2}+20 r_{24} r_{34}\right) I_{2 c}\right.} \\
\left.+2\left(r_{14}+2 r_{24}+2 r_{34}\right) J_{1 c}+6 A(3,1,1)\right\} .
\end{array}
\end{gathered}
$$

The final 14 integrals have $p_{4}=-2$ or -4 and are integrals of the third kind. We assume either that $a_{4}+b_{4} t$ is positive on the closed interval of integration or else, if $p_{4}=-2$, that it changes sign in the open interval of integration. In the latter case the integral is interpreted as a Cauchy principal value (see [3, Section 6]).

$$
\begin{aligned}
& {[1,-1,-1,-2]=I_{3 c} .} \\
& {[-1,-1,-1,-2]=\left(b_{4} I_{3 c}-b_{1} I_{1 c}\right) / d_{14} .} \\
& {[1,1,-1,-2]=\left(d_{24} I_{3 c}+b_{2} I_{2 c}\right) / b_{4} .} \\
& {[1,1,1,-2]=\left[3 b_{2} r_{24} d_{34} I_{3 c}+b_{2} b_{3}\left(r_{14}+r_{24}+r_{34}\right) I_{2 c}-J_{1 c} / b_{1}\right] / 3 b_{4} .} \\
& {[1,1,-3,-2]=\left(d_{24} I_{3 c}-J_{2 c}+d_{12} I_{1 c}\right) / d_{34} .} \\
& {[1,-1,-3,-2]=\left(b_{4} d_{23} I_{3 c}-b_{3} J_{2 c}+b_{3} d_{12} I_{1 c}\right) / d_{23} d_{34} \cdot} \\
& {[-1,-1,-3,-2]=\left[\left(b_{4}^{2} / d_{14}\right) I_{3 c}-\left(b_{3}^{2} / d_{13} d_{23}\right) J_{2 c}+\left(r_{23}^{-1}-r_{14}^{-1}\right) I_{1 c}\right] / d_{34}} \\
& {[3,-1,-1,-2]=\left(d_{14} I_{3 c}+b_{1} I_{2 c}\right) / b_{4} .} \\
& {[3,1,-1,-2]=b_{1} b_{2} r_{14} r_{24} I_{3 c}+\left(b_{1} b_{2} / 3 b_{4}\right)\left(2 r_{13}+2 r_{14}+r_{24}\right) I_{2 c}} \\
& \quad-J_{1 c} / 3 b_{3} b_{4} . \\
& {[3,1,1,-2]=\left(d_{14} d_{24} d_{34} / b_{4}^{3}\right) I_{3 c}} \\
& +\left\{b_{1} b_{2} b_{3}\left[5 r_{14}\left(r_{14}+r_{24}+r_{34}\right)-r_{12}^{2}-r_{13}^{2}-r_{23}^{2}\right] I_{2 c}\right. \\
& \left.\quad-\left(3 r_{14}+r_{24}+r_{34}\right) J_{1 c}+6 A(3,1,1)\right\} / 15 b_{4} . \\
& {[1,1,1,-4]=\left[b_{2} b_{3}\left(r_{24}+r_{34}+r_{24} r_{34} / r_{14}\right) I_{3 c}+\left(3 b_{2} b_{3} / b_{4}\right) I_{2 c}\right.} \\
& \left.-\left(d_{12} d_{13} / d_{14}\right) I_{1 c}-2 A(1,1,1,-2)\right] / 2 b_{4} .
\end{aligned}
$$

In the next three formulas we use the abbreviation

$$
K_{2 c}=b_{2} b_{3} I_{2 c}-2 b_{4} A(1,1,1,-2)=b_{3} J_{2 c}-2 d_{34} A(1,1,-1,-2)
$$


The second equality, showing that $K_{2 c}$ is finite if $x=+\infty$ or $y=-\infty$, follows from (2.17) and $[3,(4.8)]$.

$$
\begin{aligned}
& {[1,1,-1,-4]=\left[b_{1} b_{2} b_{3} b_{4}\left(r_{34}^{2}-r_{13} r_{23}\right) I_{3 c}+b_{1} r_{14} K_{2 c}\right.} \\
& \left.-d_{12} d_{13} I_{1 c}\right] / 2 d_{14} d_{34} \text {. } \\
& {[1,-1,-1,-4]=\left(1 / 2 b_{4}\right)\left(r_{14}^{-1}-r_{24}^{-1}-r_{34}^{-1}\right) I_{3 c}} \\
& +\left[K_{2 c}-\left(b_{4} d_{12} d_{13} / d_{14}\right) I_{1 c}\right] / 2 d_{24} d_{34} \text {. } \\
& {[-1,-1,-1,-4]=-\left(1 / 2 d_{14}\right)\left(r_{14}^{-1}+r_{24}^{-1}+r_{34}^{-1}\right) I_{3 c}} \\
& +\left(b_{4} / 2 d_{14} d_{24} d_{34}\right) K_{2 c} \\
& +\left(b_{1} / d_{14}\right)^{2}\left(1-r_{12} r_{13} / 2 r_{24} r_{34}\right) I_{1 c} \text {. }
\end{aligned}
$$

3. Derivation of the Formulas. Six of the 41 formulas are obtained by putting $a_{4}=1$ and $b_{4}=0$ (see the remarks following (2.15)) in suitable quartic cases in [3]. Thus $[-1,-1,-1],[1,-1,-1],[1,1,-1],[1,-1,-3],[-1,-1,-3]$, and $[1,1,-3]$ come respectively from $[-1,-1,-1,-1],[1,-1,-1,-3],[1,1,-1,-5]$, $[1,-1,-3,-3],[-1,-1,-3,-3]$, and $[1,1,-3,-3]$. Seven more are obtained by putting $a_{4}=1$ and $b_{4}=0$ and then replacing $a_{5}$ by $a_{4}$ and $b_{5}$ by $b_{4}$. Thus $[-1,-1,-1,2],[1,-1,-1,-2],[3,-1,-1,-2],[1,1,1,-4],[1,1,-1,-4],[1,-1$, $-1,-4]$, and $[-1,-1,-1,-4]$ come respectively from $[-1,-1,-1,-1,2],[1,-1$, $-1,-1,-2], \quad[3,-1,-1,-1,-2], \quad[1,1,1,-1,-4], \quad[1,1,-1,-1,-4], \quad[1,-1,-1$, $-1,-4]$, and $[-1,-1,-1,-1,-4]$. The formulas are often simplified by using identities such as $[3,(4.6)$ to $(4.9)]$.

The remaining cases are then obtained by recurrence relations. Let $e_{i}$ denote an $n$-tuple with 1 in the $i$ th place and 0 's elsewhere (for example, $\left[p+2 e_{1}\right]=$ $\left.\left[p_{1}+2, p_{2}, \ldots, p_{n}\right]\right)$. From $[4$, Section 4$]$ we have

$$
\begin{gathered}
\left(p_{1}+\cdots+p_{n}+2\right) b_{i}[p]=\sum_{j \neq i} p_{j} d_{j i}\left[p-2 e_{j}\right]+2 A\left(p+2 e_{i}\right) \\
d_{i j}[p]=b_{j}\left[p+2 e_{i}\right]-b_{i}\left[p+2 e_{j}\right] \\
b_{j}[p]=b_{i}\left[p-2 e_{i}+2 e_{j}\right]+d_{i j}\left[p-2 e_{i}\right]
\end{gathered}
$$

To get $[1,1,1]$ we use (A1) and evaluate $[1,-1,1]$ by interchanging the subscripts 2 and 3 in $[1,1,-1]$. Equations (2.28) to (2.31) then follow in order from (C12), (C13), $(\mathrm{C} 13)$, and (C13). To get $[ \pm 1,-3,-3]$ we use (B23) and evaluate $[ \pm 1,-3,-1]$ by interchanging the subscripts 2 and 3 in $[ \pm 1,-1,-3]$. Then $[-3,-3,-3]$ follows from putting $[p]=[-1,-3,-3]$ and $i=1$ in $[2,(5.5)]$ and using $[3,(4.8)]$. After $[1,-1,-5]$ has been obtained from (A2) with $[p]=[1,-1,-3]$, Eqs. (2.36) and (2.37) follow respectively from (C23) and (B13). Equation (2.38) comes from (C12).

Equations (2.40) to (2.47) follow in order from (C42), (C43), (C43), (C43), (C43), (C42), (C41), and (C42). To get (2.49), (2.50), and (2.51), we use (B14), (C24), and (C34), respectively. Equations (2.52), (2.53), (2.54), (2.56), and (2.57) follow in order from (B34), (B34), (B34), (C24), and (C14).

4. Numerical Checks. The 41 formulas in Section 2 were checked numerically when $x=2.0, y=0.5, a_{i}=0.1+0.2 i, b_{i}=0.5-0.2 i, 1 \leq i \leq 4$. In each formula the integral on the left side, defined by $(2.20)$, was integrated numerically by the SLATEC code QNG. On the right-hand side, $I_{1 c}, I_{2 c}$, and $I_{3 c}$ were calculated from 
(2.12) to (2.14) by using the codes for $R$-functions in the Supplements to [2] and [3]. The remaining calculations, including those of $J_{1 c}, J_{2 c}$, and $K_{2 c}$ by (2.16), (2.17), and (2.59), were done with a hand calculator. For each of the 41 formulas the values obtained for the two sides agreed to better than one part in a million.

Some of the intermediate values in these calculations are listed here:

$$
\begin{array}{ll}
U_{1}^{2}=0.41309998, & W_{2}^{2}=0.38909998, \\
U_{2}^{2}=0.40109998, & P_{2}^{2}=0.24016665, \\
U_{3}^{2}=0.43709998, & Q_{2}^{2}=0.21616665, \\
& \\
R_{C}\left(P_{2}^{2}, Q_{2}^{2}\right)=2.1128946, & I_{1 c}=3.0973715, \\
R_{F}\left(U_{3}^{2}, U_{2}^{2}, U_{1}^{2}\right)=1.5486858, & I_{2 c}=2.0520132, \\
R_{D}\left(U_{3}^{2}, U_{2}^{2}, U_{1}^{2}\right)=3.7353179, & I_{3 c}=4.2877248, \\
R_{J}\left(U_{3}^{2}, U_{2}^{2}, U_{1}^{2}, W_{2}^{2}\right)=3.8709720, & J_{1 c}=-0.00688951, \\
& J_{2 c}=-0.80566308, \\
A(1,1,1)=0.16015635, & K_{2 c}=0.78110328, \\
A(1,1,-1)=0.50543220, & \\
A(1,-1,-1)=0.48163106, & A(3,1,1)=0.32463223, \\
A(-1,1,-1)=-0.12403646, & A(1,1,1,-2)=1.3360390, \\
A(1,1,-3)=1.2956636, & A(1,1,-1,-2)=2.9189040 .
\end{array}
$$

Ames Laboratory and Department of Mathematics Iowa State University

Ames, Iowa 50011

E-mail: s1.mth@isumvs.bitnet

1. P. F. BYRD \& M. D. FRIEDMAN, Handbook of Elliptic Integrals for Engineers and Scientists, 2nd ed., Springer-Verlag, New York, 1971.

2. B. C. CARLSON, "A table of elliptic integrals of the second kind," Math. Comp., v. 49, 1987, pp. 595-606. (Supplement, ibid., S13-S17.)

3. B. C. CARLSON, "A table of elliptic integrals of the third kind," Math. Comp., v. 51, 1988, pp. 267-280. (Supplement, ibid., S1-S5.)

4. I. S. GRAdShteyn \& I. M. RYzhik, Table of Integrals, Series, and Products, Academic Press, New York, 1980.

5. A. P. Prudnikov, Yu. A. BRychkov \& O. I. MaricheV, Integrals and Series, vol. 1, Gordon and Breach, New York, 1986. 